\title{
The role of adjuvant external beam radiation therapy for papillary thyroid carcinoma invading the trachea
}

Young Suk Kim, MD', Jae Hyuck Choi, MD², Kwang Sik Kim, MD², Gil Chae Lim, MD², Jeong Hong Kim, MD², Ju Wan Kang, $\mathrm{MD}^{3}$, Hee-Sung Song, MD ${ }^{4}$, Sang Ah Lee, $\mathrm{MD}^{5}$, Chang Lim Hyun, MD, PhD ${ }^{6}$, Yunseon Choi, MD, PhD', Gwi Eon Kim, MD, PhD ${ }^{1}$

Departments of ${ }^{1}$ Radiation Oncology, ${ }^{2}$ Surgery, ${ }^{3}$ Otorhinolaryngology, ${ }^{4}$ Nuclear Medicine and Molecular Imaging, ${ }^{5}$ Internal Medicine, and ${ }^{6}$ Pathology, Jeju National University Hospital, Jeju National University School of Medicine, Jeju; ${ }^{7}$ Department of Radiation Oncology, Inje University Busan Paik Hospital, Inje University College of Medicine, Busan, Korea

Purpose: To evaluate the effect of adjuvant external beam radiation therapy (EBRT) on local failure-free survival rate (LFFS) for papillary thyroid cancer (PTC) invading the trachea.

Materials and Methods: Fifty-six patients with locally advanced PTC invading the trachea were treated with surgical resection. After surgery, 21 patients received adjuvant EBRT and radioactive iodine therapy (EBRT group) and 35 patients were treated with radioactive iodine therapy (control group).

Results: The age range was 26-87 years (median, 56 years). The median follow-up period was 43 months (range, 4 to 145 months). EBRT doses ranged from 50.4 to $66 \mathrm{~Gy}$ (median, $60 \mathrm{~Gy}$ ). Esophagus invasion and gross residual disease was more frequent in the EBRT group. In the control group, local recurrence developed in $9(9 / 35,26 \%)$ and new distant metastasis in $2(2 / 35,6 \%)$ patients, occurring 4 to 68 months (median, 37 months) and 53 to 68 months (median, 60 months) after surgery, respectively. Two patients had simultaneous local recurrence and new distant metastasis. There was one local failure in the EBRT group at 18 months after surgery $(1 / 21,5 \%)$. The 5 -year LFFS was $95 \%$ in the EBRT group and $63 \%$ in the control group $(p=0.103)$. In the EBRT group, one late grade 2 xerostomia was developed.

Conclusion: Although, EBRT group had a higher incidence of esophagus invasion and gross residual disease, EBRT group showed a better 5 -year LFFS. Adjuvant EBRT may have contributed to the better LFFS in these patients.

Keywords: Thyroid carcinoma, Papillary, Radiotherapy, Adjuvant, Trachea

\section{Introduction}

For most patients with differentiated thyroid cancer, surgery, radioactive iodine therapy (RAI), and thyroid stimulating hormone (TSH) suppression are effective in achieving locoregional control [1]. The role of adjuvant external beam radiation therapy (EBRT) in differentiated thyroid cancer is debated because of a lack of prospective data, as well

Received 10 April 2017, Revised 04 May 2017, Accepted 19 May 2017.

Correspondence: Gwi Eon Kim, MD, PhD, Department of Radiation Oncology, Jeju National University Hospital, Jeju National University School of Medicine, 15 Aran 13-gil, Jeju 63241, Korea. Tel: +82-64-717-1330, Fax: +82-64-717-1169, E-mail: gekim@ yuhs.ac

Abstract accepted for the 57th Annual Meeting of the American Society for Radiation Oncology, San Antonio, TX, USA on October 18-21, 2015.

(c) This is an Open Access article distributed under the terms of the Creative Commons Attribution Non-Commercial License (http://creativecommons.org/ licenses/by-nc/4.0/) which permits unrestricted non-commercial use, distribution, and reproduction in any medium, provided the original work is properly cited.

www.e-roj.org 
as inhomogeneity and conflicting results in the existing retrospective data [1-6]. However, it has been a challenging obstacle to treat patients with local invasion or encasement of surrounding structures (e.g., trachea, esophagus, thyroid cartilage, recurrent laryngeal nerve, strap muscle, mediastinum, or great vessels) $[3,4,7]$. High local recurrence and mortality rate are usually observed in these patients $[5,8]$. To reduce inhomogeneity in our study population, we focused on patients with a high risk of local recurrence, i.e., those with trachea-invading papillary thyroid carcinoma (PTC) who have undergone curative surgery. We evaluated whether adjuvant EBRT affects the local failure-free survival rate (LFFS) in patients with PTC invading the trachea.

\section{Materials and Methods}

\section{Patients}

The medical records of 951 patients with PTC who underwent thyroid surgery at two institutes between May 2003 and January 2017 were retrospectively reviewed. Our hospital Institutional Review Board approved this study. Sixty-five $(65 / 951,7 \%)$ patients were found to have tracheal cartilage invasion. Nine patients were excluded from the analysis because one patient had distant metastasis at initial diagnosis, RAI was not delivered after thyroid surgery in 2 patients, neck dissection was not performed in 4 patients, one patient received adjuvant EBRT with palliative intent after palliative surgery, and adjuvant EBRT was stopped at 30 Gy due to suspicion of Stevens-Johnson syndrome, a form of toxic epidermal necrolysis, in 1 patient. Patients referred with recurrent disease were excluded [9]. Thus, data from a total of 56 patients were analyzed. Tracheal invasion was confirmed by ultrasound, computed tomography (CT), magnetic resonance imaging (MRI), gross and microscopic pathologic findings, surgical findings, or a combination of these. After surgery, 21 patients received adjuvant EBRT and RAI (the EBRT group), and 35 patients were treated with RAl alone (the control group). Adjuvant EBRT was recommended on the basis of surgical findings of gross residual disease in 5 patients and concern of microscopic residual disease despite visibly complete resection in 16 patients. The clinical stage was determined according to the American Joint Committee on Cancer staging system, 7th edition. All cases had a stage T4a tumor extending beyond the thyroid capsule to invade subcutaneous soft tissues, larynx, trachea, esophagus, or recurrent laryngeal nerve [3]. The majority of patients with tracheal invasion showed Types I or II (Type I, cancer that extended through the capsule and abutted the external perichondrium but that did not erode the cartilage; Type II, cancer that invaded between the rings of cartilage or destroyed the cartilage), as described by Shin et al. $[3,10]$.

The medical records of patients were retrospectively reviewed to collect the following data: demographic information such as gender and age at diagnosis; imaging studies including chest X-ray, thyroid ultrasound, 131I wholebody scan, neck CT, MRI, and positron emission tomography; operation records including extent of surgery and invasion to adjacent organs; various histological parameters including primary tumor size, extrathyroidal extension, resection margin status, and cervical lymph node involvement; distant metastasis; RAI therapy and accumulated RAI doses; EBRT; and clinical outcome at the last follow-up. For multifocal tumors, the diameter of the largest tumor was taken as the primary tumor size [11].

\section{Surgery}

After imaging studies and before EBRT, all patients underwent surgery. All 56 patients underwent total thyroidectomy with central neck dissection. Modified radical neck dissection was performed in 9 patients $(9 / 56,16 \%)$. For locally extensive T4 disease, maximal surgical resection was performed, i.e., the tumor was shaved off the trachea or adjacent structures [12]. The surgical margin status and completeness of resection (RO, uninvolved margins; R1, microscopically involved margins/no gross residual disease; or R2, involved margins/gross residual disease) were determined by reviewing the operative notes and pathology report for each case [13].

\section{External beam radiation therapy}

Fifteen patients $(15 / 21,71 \%)$ started EBRT between 4 and 5 weeks after surgery, and 100\% had begun EBRT by 3 months. Patients were treated using thermoplastic immobilization masks to ensure adequate immobilization during therapy and reproducibility. Treatment was conducted using a linear accelerator with the Millennium 120-leaf multi-leaf collimators system (Clinac iX; Varian Medical Systems Inc., Palo Alto, CA, USA). Patient set-up was verified weekly by $\mathrm{kV}$ portal images obtained using the On-Board Imager system prior to treatment. The majority of patients were treated with volumetric modulated arc therapy or 7-field fixed intensitymodulated radiation therapy (IMRT) using a simultaneous integrated boost with 1.8-2.2 Gy per fraction.

The gross tumor volume (GTV) included the tracheal wall invasion of the tumor and any gross residual tumor. A 1-2 

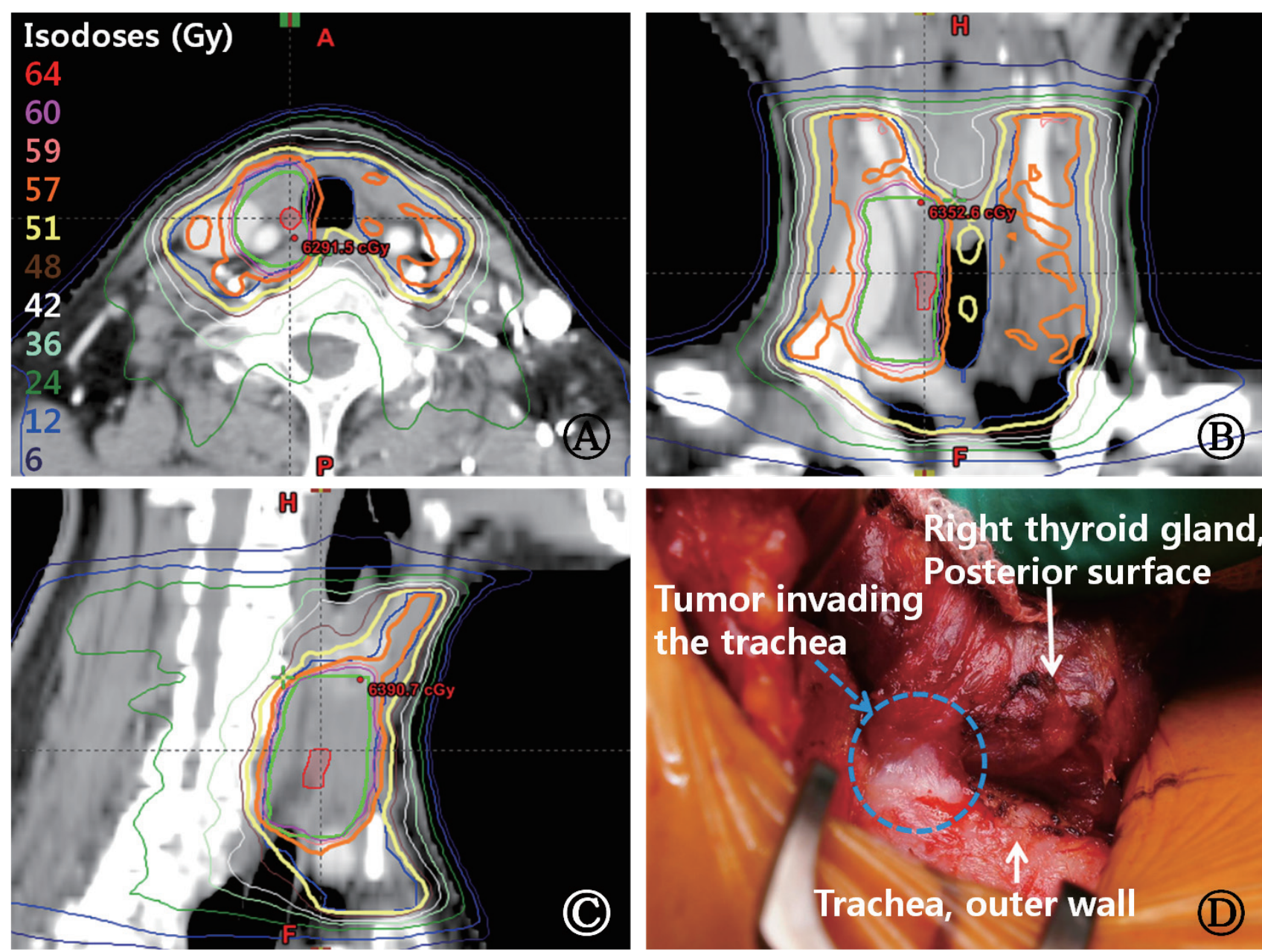

Fig. 1. Representative intensity-modulated radiotherapy treatment plan (A-C) for a 64-year-old female with papillary thyroid carcinoma invading the tracheal outer layer (D), initially treated using total thyroidectomy and right central neck dissection. (D) Intra-operative photograph. The primary tumor was a $0.8-\mathrm{cm}$ mass. Preoperative neck computed tomography and thyroid ultrasound findings revealed no enlarged neck lymph nodes. Three metastatic lymph nodes were found among five dissected neck lymph nodes without extracapsular extension in the postoperative pathologic findings. The patient received postoperative radiotherapy because of a close resection margin (less than $1 \mathrm{~mm}$ ) after shaving off the trachea outer wall, staged pT4a N1 MO. The patient received 60 Gy to the high-risk CTV (green) encompassing the surgical bed directly involved with gross disease (red), 54 Gy to the intermediate-risk CTV (blue) encompassing adjacent soft tissues and right-sided central neck dissection bed and bilateral neck levels III, IV, and VI. All doses were delivered over 30 daily fractions. CTV, clinical target volume.

$\mathrm{cm}$ margin was added to the GTV for microscopic extension, and this was considered the high-risk clinical target volume (CTV1). CTV1 included an operative bed involving a close margin, positive margin, or gross residual disease. The low- to intermediate-risk CTV (CTV2) included positive regional lymph nodes located in the region from the hyoid bone to the sternal notch (neck levels III, IV, and VI on both sides). It sometimes included neck levels II and $V$ on both sides. A planning target volume (PTV) was generated by expanding the CTV by $0.3 \mathrm{~cm}$ in all directions. Generally, PTV1 was treated with 50.4-66 Gy and
PTV2 was treated with 50.4-54 Gy in 28-33 daily fractions. Fig. 1 depicts typical target delineation and isodose curves.

\section{Radioactive iodine therapy}

RAl was delivered after primary thyroid surgery in both groups ( $n=56,100 \%$ ). The patients received RAI at a median 4 months (range, 1 to 10 months) after primary surgery. In the EBRT group, RAI was performed before adjuvant EBRT in 4 patients and after adjuvant EBRT in 17 patients. Fifty-three patients received one dose, 2 patients received two doses, and 
Table 1. Patient characteristics

\begin{tabular}{|c|c|c|c|}
\hline & $\operatorname{EBRT}(n=21)$ & Control $(n=35)$ & $p$-value \\
\hline Follow-up (mo) & $44(6-65)$ & $43(4-145)$ & \\
\hline Age (yr) & $54(36-87)$ & $56(26-83)$ & \\
\hline$<45$ (younger) & $2(10)$ & $6(17)$ & 0.696 \\
\hline$\geq 45$ & $19(90)$ & $29(83)$ & \\
\hline \multicolumn{4}{|l|}{ Gender } \\
\hline Male & $4(19)$ & $7(20)$ & 1.000 \\
\hline Female & $17(81)$ & $28(80)$ & \\
\hline Tumor size $(\mathrm{cm})$ & $1.2(0.5-6.0)$ & $1.3(0.2-4.0)$ & 0.372 \\
\hline \multicolumn{4}{|l|}{ Tumor multiplicity } \\
\hline Yes & $9(43)$ & $16(46)$ & 0.835 \\
\hline No & $12(57)$ & $19(54)$ & \\
\hline \multicolumn{4}{|l|}{ Esophagus invasion } \\
\hline Yes & $6(29)$ & $1(3)$ & 0.009 \\
\hline No & $15(71)$ & $34(97)$ & \\
\hline \multicolumn{4}{|l|}{ Recurrent laryngeal nerve invasion } \\
\hline Yes & $7(33)$ & $6(17)$ & 0.165 \\
\hline No & $14(67)$ & $29(83)$ & \\
\hline \multicolumn{4}{|l|}{ Regional node stage } \\
\hline No & $4(19)$ & $10(29)$ & 0.532 \\
\hline N1 & $17(81)$ & $25(71)$ & \\
\hline \multicolumn{4}{|l|}{ Resection margin } \\
\hline Negative (R0) & $7(33)$ & $16(46)$ & 0.092 \\
\hline Microscopic residual disease (R1) & $7(33)$ & $11(31)$ & \\
\hline Gross residual disease (R2) & $5(24)$ & $1(3)$ & \\
\hline Probable R1 & $2(10)$ & $7(20)$ & \\
\hline
\end{tabular}

Values are presented as median (range) or number (\%).

EBRT, external-beam radiation therapy.

one received three doses of RAI. The median total dose of RAI was $150 \mathrm{mCi}(5.55 \mathrm{GBq}$ ) (range, 30 to $450 \mathrm{mCi}$ ). A whole-body scan was performed 1 week after 131I administration [14]. Success of the ablation was documented by a negative scan. Repeated doses were given if the scan showed uptake [15]. Levothyroxine treatment was then initiated in all patients to reduce the TSH level [14].

\section{Treatment response}

Local failure was defined as clinical or radiographic increase in the size of primary tumor or regional lymph nodes. In some cases, recurrence was demonstrated pathologically by surgical resection or biopsy [16]. LFFS was defined as the time interval from the date of primary surgery to the date of local failure, death, or last contact, as appropriate. Distant metastasis was defined as any recurrence outside of the cervical neck and the upper mediastinum [16].

Patients were followed up by an endocrinologist, thyroid surgeon, and/or radiation oncologist every 3 months for the first 2 postoperative years, then every 6 months for up to 5 years, and annually thereafter [16]. Follow-up assessment included a physical examination, serum thyroglobulin level, chest X-ray, neck ultrasound and/or CT, 131I whole-body scan, and other radiologic studies, if necessary [3]. Most patients underwent fine needle aspiration cytology or excision biopsy of suspicious neck masses to confirm recurrence [17].

Patients were evaluated weekly during EBRT. Acute toxicity was reported during both treatment and follow-up visits. Acute side effects were defined as those occurring within 3 months of radiotherapy completion. Information regarding both acute and late toxicity was collected from the clinical visit documentation. All toxicity was rated using the National Cancer Institute Common Terminology Criteria for Adverse Events v3.0 [18].

\section{Statistical analysis}

Statistical analysis was performed using IBM SPSS ver. 22.0 software package (IBM SPSS Inc., Armonk, NY, USA). The distributions of factors among the EBRT and control groups were compared using the Fisher exact test and chi-square test. The Kaplan-Meier method was used to determine the LFFS. The distributions of LFFS were compared using the log-rank test. Statistical significance was defined as a $p$-value of $<0.05$. Age refers to the patient's age at the time of diagnosis [19]. 


\section{Results}

\section{Patient characteristics}

The baseline characteristics of patients are listed in Table 1. The percentages of patients with esophagus invasion and gross residual disease were higher in the EBRT group than in the control group. Baseline characteristics including age, gender, tumor size, status of lymph node metastasis, and extent of surgery did not differ significantly between groups. The age range was 26-87 years (median, 56 years). The median followup period was 43 months (range, 4 to 145 months) for all patients. All patients were followed up until death or to the time this article was written, except 2 patients.

The median total radiotherapy dose was 60 Gy (range, 50.4 to $66 \mathrm{~Gy}$ ) administered in 5 daily fractions per week. The median radiation dose delivered to patients with gross residual disease was 63 Gy (range, 60 to 64 Gy). The median radiation dose delivered to patients without gross residual disease was $60 \mathrm{~Gy}$ (range, 50.4 to $66 \mathrm{~Gy}$ ).

\section{Treatment outcome}

At the time of analysis, all patients $(n=56)$ were alive 4 to 145 months after surgery (median, 43 months). In the control group, local recurrence developed in $9(9 / 35,26 \%)$ and new distant metastasis in 2 patients $(2 / 35,6 \%) 4$ to 68 months (median, 37 months) and 53 to 68 months (median, 60 months) after surgery, respectively (Fig. 2). Two patients had simultaneous local recurrence and new distant metastasis. The sites of newly developed distant metastases included lung $(n=2)$ and bone $(n=1)$. There was one local failure in the EBRT group at 18

\section{Control}

Local failure Regional failure $(11 \%)$

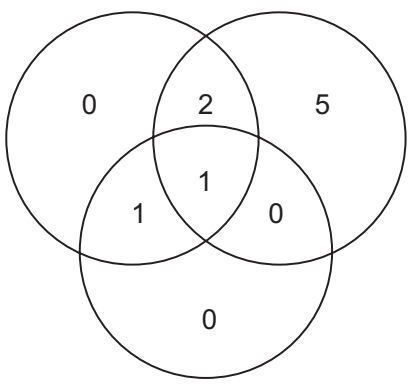

Distant metastasis

$(6 \%)$

\section{EBRT}

Local failure Regional failure $(5 \%)$

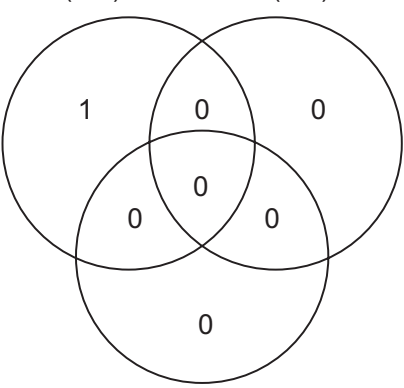

Distant metastasis $(0 \%)$
Fig. 2. Patterns of treatment failure. EBRT, external-beam radiation therapy. months after surgery $(1 / 21,5 \%)$.

The median LFFS in the EBRT and control groups was 44 months (range, 6 to 65 months) and 43 months (range, 4 to 85 months), respectively. The 5 -year LFFS rate was $95 \%$ in the EBRT group and 63\% in the control group $(p=0.103)$ (Fig. 3).

Of 10 patients who experienced local recurrence, repeated operations were performed in 6 patients (Table 2). Severe surgical morbidity was not found. One patient complained about left lateral neck pain and discomfort after salvage operation for 1 year. Local recurrences developed mainly in the thyroid tumor bed and ipsilateral neck node area. In Case 3, right thyroid tumor bed recurrence developed 45 months after initial operation. The patient received salvage EBRT for tumor bed recurrence with a total dose of $66 \mathrm{~Gy}$ in 30 fractions, given as 5 daily fractions per week. The tumor bed recurrent lesion disappeared. New distant metastases (right upper lung single 6-mm nodule and sacrum) developed 7 months after salvage EBRT. Surgical resection for the lung nodule was recommended to the patient, but he refused the surgery. Although there was no significant difference in tumor size between the two groups, five recurrent cases in the control group had tumor sizes larger than $2 \mathrm{~cm}$ (case \#1, \#2,\#4,\#6, and \#8) (Table 2).

\section{Complications after external beam radiation therapy} Incidences of acute and late toxicity in the EBRT group are shown in Table 3. None of the patients in the EBRT group developed acute or late toxicities higher than grade 2. None of the patients required short-term percutaneous endoscopic gastrostomy.

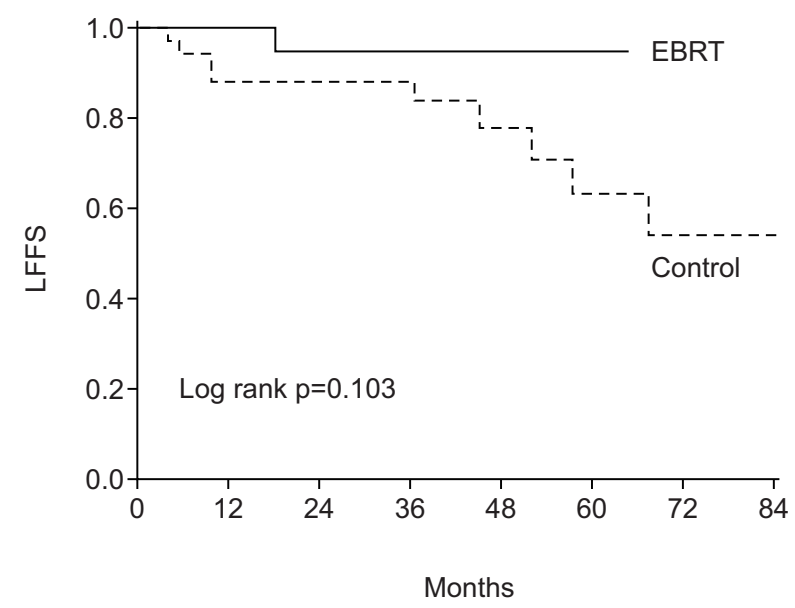

Fig. 3. Local failure-free survival (LFFS) rate in the external-beam radiation therapy (EBRT) and control groups. 


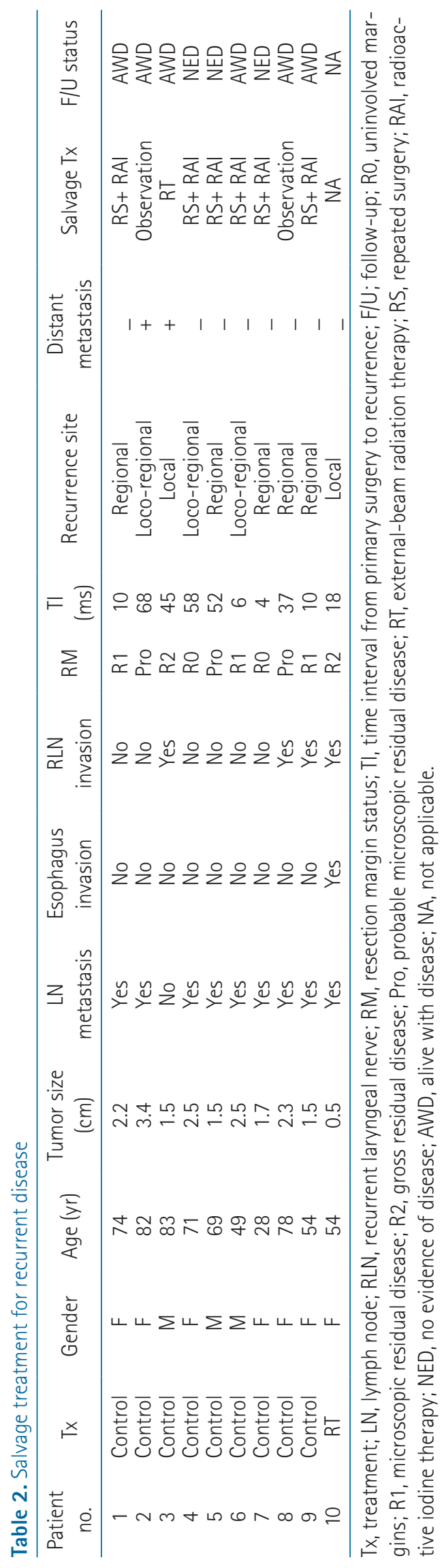

Table 3. Incidence of acute and late toxicity in the EBRT group $(n=21)$

\begin{tabular}{lc}
\hline \multicolumn{1}{c}{ Complication } & No. of patients (\%) \\
\hline Acute (within 3 mo) & \\
Dysphagia & \\
Grade 1 & $20(95)$ \\
Grade 2 & $1(5)$ \\
Dermatitis & $20(95)$ \\
Grade 1 (erythema) & $4(19)$ \\
Grade 1 (dry desquamation) & $1(5)$ \\
Late (>3 mo) & $1(5)$ \\
Xerostomia Grade 2 & $1(5)$ \\
Parotitis with pain Grade 1 & \\
Hyperpigmentation Grade 1 & \\
\hline EBRT, external-beam radiation therapy. &
\end{tabular}

\section{Discussion and Conclusion}

Several institutions have reported outcomes of PTC patients treated with EBRT $[4,6,7,20-27]$. Irradiation was generally used for patients in whom excision had been especially difficult and whose prognosis appeared to be less favorable [20]. Indications for adjuvant EBRT generally include high-risk presentations involving local invasion or encasement of surrounding structures (e.g., trachea, esophagus, thyroid cartilage, recurrent laryngeal nerve, strap muscle, mediastinum, or great vessels) with evidence of residual microscopic or gross disease $[17,23]$. In this study, we evaluated the LFFS between the EBRT and control groups for patients with locally advanced PTC invading the trachea that was treated with curative surgery. The 5-year LFFS rate was 95\% in the EBRT group and 63\% in the control group $(p=0.103)$. There may be selection bias in the EBRT group, given that most patients were referred because of concerns regarding the adequacy of local control following surgery alone. Those treated with adjuvant EBRT had a higher incidence of adverse baseline risk factors, especially esophagus invasion and gross residual disease (Table 1).

Farahati et al. [6] reported that non-irradiated patients with advanced PTC (stage pT4) tended to have frequent recurrences. Adjuvant EBRT, in contrast, limited this tendency significantly in the irradiated group of patients $(p=0.0001)$. Chow et al. [15] also reported that adjuvant EBRT improved locoregional control in 124 PTC patients with gross residual disease in the neck ( $p=0.0019$ ). Keum et al. [3] analyzed the effect of adjuvant EBRT on patients treated with 'shave' excision of the tracheal cartilage for PTC invading the trachea. The 10-year LFFS rate of the EBRT group was significantly better than that of the control group (89\% in the EBRT group vs. $38 \%$ in the control group) $(p<0.01)$. 
The optimal adjuvant radiation dose is not known. In most previous studies of PTC patients, the radiation dose ranged from 60 Gy to 72 Gy with conventional fraction sizes $[4,6,15,20,23,24]$. In this study, the radiation dose ranged from $50.4 \mathrm{~Gy}$ to $66 \mathrm{~Gy}$ (median, $60 \mathrm{~Gy}$ ). Schwartz et al. [23] described their prescription of the following doses: the operative bed is treated to adjuvant doses of 57-60 Gy, microscopic positive surgical margins can be boosted to doses of $63 \mathrm{~Gy}$, and gross disease is treated to doses of 66-70 Gy. Similar radiation dose recommendations were recently published by the American Head and Neck Society [1]. Additional investigation is necessary to define the optimal curative dose prescriptions for PTC [23].

Locoregionally recurrent disease is managed similarly to locally advanced disease at initial presentation [23]. Many authors reported the usefulness of EBRT in the treatment of recurrent PTC patients $[7,18,23,25,28]$. In this study, we did not include the patients with recurrent disease in the study cohort, for homogeneity. One patient in the control group recurred in the thyroid tumor bed. The patient was treated with salvage EBRT without repeated operation (Table 2).

One major reason for avoiding EBRT in patients with PTC is the toxicity associated with this therapeutic modality. The acute toxicities reported include oral mucositis, taste changes, xerostomia, pharyngitis, laryngitis, dysphagia, hoarseness, and skin reactions (early erythema, and dry or moist desquamation) $[23,29]$. Late complications include fibrosis and atrophy of the neck muscles and soft tissue, tracheal and esophageal stenosis, chronic dysphagia, chronic laryngeal edema, xerostomia, and radiation pneumonitis [23,30-32]. In this study, EBRT complications were not uncommon, but severe complications were rare (Table 3). One possible reason is that 20 patients (95\%) were treated with the IMRT technique, sparing some critical organs (submandibular glands, soft tissues, mandible, esophagus, hypopharynx, and vocal cords). It is well known that IMRT is superior to 3-dimensional conformal radiotherapy for normal organ dose sparing [23]. Another reason is that the treatment volume was relatively smaller than other EBRT studies $[4,6,17,23,24]$. Relatively small tumor size (median, 1.2 $\mathrm{cm}$ ) may also have contributed to the less severe complications [23] (Table 1).

This study has several limitations. As with other retrospective analyses, the influence of patient selection bias needs to be considered in the interpretation of any results $[9,19]$. Eight of 35 patients treated with surgery and RAI (the control group, 23\%) were historical controls that had been treated several years before the EBRT group (median, 2 years; range, 1 to 6 years), because our institution's EBRT facility was equipped in August 2009. The other limitation of our study was the short follow-up period. In many series with a long follow-up, authors observed some late recurrences [20]. Longer follow-up is needed to assess the full impact of EBRT on local recurrence, survival, and late complications. Another limitation was the small number of patients.

In conclusion, although, EBRT group had a higher incidence of adverse risk factors, especially esophagus invasion and gross residual disease, EBRT group showed a better five-year LFFS. Adjuvant EBRT may have contributed to the better LFFS in these patients. Randomized studies are needed to confirm the benefit of adjuvant EBRT in trachea invading patients.

\section{Conflict of Interest}

No potential conflict of interest relevant to this article was reported.

\section{Acknowledgments}

This work was supported by the research grant of the Jeju National University Hospital.

\section{References}

1. Kiess AP, Agrawal N, Brierley JD, et al. External-beam radiotherapy for differentiated thyroid cancer locoregional control: a statement of the American Head and Neck Society. Head Neck 2016;38:493-8.

2. Samaan NA, Schultz PN, Hickey RC, et al. The results of various modalities of treatment of well differentiated thyroid carcinomas: a retrospective review of 1599 patients. J Clin Endocrinol Metab 1992;75:714-20.

3. Keum KC, Suh YG, Koom WS, et al. The role of postoperative external-beam radiotherapy in the management of patients with papillary thyroid cancer invading the trachea. Int J Radiat Oncol Biol Phys 2006;65:474-80.

4. Kim TH, Yang DS, Jung KY, Kim CY, Choi MS. Value of external irradiation for locally advanced papillary thyroid cancer. Int J Radiat Oncol Biol Phys 2003;55:1006-12.

5. Lin JD, Tsang NM, Huang MJ, Weng HF. Results of external beam radiotherapy in patients with well differentiated thyroid carcinoma. Jpn J Clin Oncol 1997;27:244-7.

6. Farahati J, Reiners C, Stuschke M, et al. Differentiated thyroid cancer. Impact of adjuvant external radiotherapy in patients with perithyroidal tumor infiltration (stage pT4). Cancer 
1996;77:172-80.

7. Kwon J, Wu HG, Youn YK, Lee KE, Kim KH, Park DJ. Role of adjuvant postoperative external beam radiotherapy for well differentiated thyroid cancer. Radiat Oncol J 2013;31:162-70.

8. Mazzaferri EL, Jhiang SM. Long-term impact of initial surgical and medical therapy on papillary and follicular thyroid cancer. Am J Med 1994:97:418-28.

9. Brierley J, Tsang R, Panzarella T, Bana N. Prognostic factors and the effect of treatment with radioactive iodine and external beam radiation on patients with differentiated thyroid cancer seen at a single institution over 40 years. Clin Endocrinol (0xf) 2005;63:418-27.

10. Shin DH, Mark EJ, Suen HC, Grillo HC. Pathologic staging of papillary carcinoma of the thyroid with airway invasion based on the anatomic manner of extension to the trachea: a clinicopathologic study based on 22 patients who underwent thyroidectomy and airway resection. Hum Pathol 1993;24:866-70.

11. Kim HJ, Kim NK, Choi JH, et al. Radioactive iodine ablation does not prevent recurrences in patients with papillary thyroid microcarcinoma. Clin Endocrinol (Oxf) 2013;78:614-20.

12. Chow SM, Yau S, Kwan CK, Poon PC, Law SC. Local and regional control in patients with papillary thyroid carcinoma: specific indications of external radiotherapy and radioactive iodine according to $\mathrm{T}$ and $\mathrm{N}$ categories in AJCC 6th edition. Endocr Relat Cancer 2006;13:1159-72.

13. Nelson JW, Ghafoori AP, Willett CG, et al. Concurrent chemoradiotherapy in resected extrahepatic cholangiocarcinoma. Int J Radiat Oncol Biol Phys 2009;73:148-53.

14. Liou MJ, Tsang NM, Hsueh C, Chao TC, Lin JD. Therapeutic outcome of second primary malignancies in patients with well-differentiated thyroid cancer. Int J Endocrinol 2016;2016:9570171.

15. Chow SM, Law SC, Mendenhall WM, et al. Papillary thyroid carcinoma: prognostic factors and the role of radioiodine and external radiotherapy. Int J Radiat Oncol Biol Phys 2002;52:784-95.

16. Lee EK, Lee YJ, Jung YS, et al. Postoperative simultaneous integrated boost-intensity modulated radiation therapy for patients with locoregionally advanced papillary thyroid carcinoma: preliminary results of a phase II trial and propensity score analysis. J Clin Endocrinol Metab 2015;100:1009-17.

17. Azrif M, Slevin NJ, Sykes AJ, Swindell R, Yap BK. Patterns of relapse following radiotherapy for differentiated thyroid cancer: implication for target volume delineation. Radiother Oncol 2008;89:105-13.
18. Terezakis SA, Lee KS, Ghossein RA, et al. Role of external beam radiotherapy in patients with advanced or recurrent nonanaplastic thyroid cancer: Memorial Sloan-kettering Cancer Center experience. Int J Radiat Oncol Biol Phys 2009;73:795-801.

19. Mazzaferri EL, Jhiang SM. Differentiated thyroid cancer longterm impact of initial therapy. Trans Am Clin Climatol Assoc 1995;106:151-70.

20. Tubiana $M$, Haddad $E$, Schlumberger $M$, Hill $C$, Rougier $P$, Sarrazin D. External radiotherapy in thyroid cancers. Cancer 1985;55(9 Suppl):2062-71.

21. Simpson WJ, Panzarella T, Carruthers JS, Gospodarowicz MK, Sutcliffe SB. Papillary and follicular thyroid cancer: impact of treatment in 1578 patients. Int J Radiat Oncol Biol Phys 1988;14:1063-75.

22. Tsang RW, Brierley JD, Simpson WJ, Panzarella $T$, Gospodarowicz MK, Sutcliffe SB. The effects of surgery, radioiodine, and external radiation therapy on the clinical outcome of patients with differentiated thyroid carcinoma. Cancer 1998;82:375-88.

23. Schwartz DL, Lobo MJ, Ang KK, et al. Postoperative external beam radiotherapy for differentiated thyroid cancer: outcomes and morbidity with conformal treatment. Int J Radiat Oncol Biol Phys 2009;74:1083-91.

24. Meadows KM, Amdur RJ, Morris CG, Villaret DB, Mazzaferri EL, Mendenhall WM. External beam radiotherapy for differentiated thyroid cancer. Am J Otolaryngol 2006;27:24-8.

25. O'Connell ME, A'Hern RP, Harmer CL. Results of external beam radiotherapy in differentiated thyroid carcinoma: a retrospective study from the Royal Marsden Hospital. Eur J Cancer 1994;30A:733-9.

26. Wu XL, Hu YH, Li QH, et al. Value of postoperative radiotherapy for thyroid cancer. Head Neck Surg 1987;10:107-12.

27. Esik O, Németh G, Eller J. Prophylactic external irradiation in differentiated thyroid cancer: a retrospective study over a $30-$ year observation period. Oncology 1994;51:372-9.

28. Lee $N$, Tuttle M. The role of external beam radiotherapy in the treatment of papillary thyroid cancer. Endocr Relat Cancer 2006;13:971-7.

29. Kim JH, Jenrow KA, Brown SL. Mechanisms of radiationinduced normal tissue toxicity and implications for future clinical trials. Radiat Oncol J 2014;32(3):103-15.

30. Kim J, Shin ES, Kim JE, Yoon SP, Kim YS. Neck muscle atrophy and soft-tissue fibrosis after neck dissection and postoperative radiotherapy for oral cancer. Radiat Oncol J 2015;33:344-9.

31. Sun XS, Sun SR, Guevara N, et al. Indications of external beam radiation therapy in non-anaplastic thyroid cancer and impact 
Young Suk Kim, et al

of innovative radiation techniques. Crit Rev Oncol Hematol 2013;86:52-68.

32. Biermann M, Pixberg M, Riemann B, et al. Clinical outcomes of adjuvant external-beam radiotherapy for differentiated thyroid cancer: results after 874 patient-years of follow-up in the MSDS-trial. Nuklearmedizin 2009;48:89-98. 